\title{
Safety of Lateral Interbody Fusion Surgery without Intraoperative Monitoring
}

\author{
Hong-Jae LEE ${ }^{1}$, Kyeong-Sik RYU², Jung-Woo HUR ${ }^{2}$, Ji-Hoon SEONG², Hyun-Jin $\mathrm{CHO}^{2}$, Jin-Sung KIM² \\ ${ }^{1}$ Catholic University of Korea, College of Medicine, Daejeon St. Mary's Hospital, Department of Neurosurgery, Daejeon, Korea \\ ${ }^{2}$ Catholic University of Korea, College of Medicine, Seoul St. Mary's Hospital, Department of Neurosurgery, Seoul, Korea \\ This study was presented at International Society for the Advancement of Spine Surgery (ISASS) meeting in April 12-14, 2017.
}

\section{ABSTRACT}

AIM: Oblique lateral interbody fusion (OLIF) is a minimally invasive surgical method that can provide an access to the lumbar spine without direct dissection of psoas muscle and the need for intraoperative neuromonitoring (IOM) is questionable. The aim of this study was to examine and document the transient and persistent perioperative complications in patients who underwent OLIF for degenerative lumbar disease without IOM.

MATERIAL and METHODS: A total of 129 consecutive patients who were diagnosed as degenerative spinal disease from L1 to S1 and underwent mini-open OLIF were identified and retrospectively reviewed. All patients were classified as two groups; non-IOM group and IOM group. According to the relation to surgical procedure, the complications were divided into two groups; 'procedurerelated' and 'procedure-unrelated'. Based on the effect of duration, the complications were defined as 'transient' where the symptom is relieved within 30 days postoperatively, and 'persistent' where the symptom remains for more than 30 days postoperatively.

RESULTS: The study groups comprised 57 cases in the IOM group and 72 in the non-IOM group. The complication rate was $24.6 \%$ (transient; $17.6 \%$, persistent; $7.0 \%$ ) in the IOM group and $29.2 \%$ (transient; $25.0 \%$, persistent; $4.2 \%$ ) in the non-IOM group. The incidence of postoperative leg symptoms related to lumbar plexus and/or psoas muscle injury was 6 transient and 3 persistent in the IOM group (overall 15.3\%), and 12 transient and 3 persistent in the non-IOM group (overall 20.9\%).

CONCLUSION: Mini-open OLIF can be safely carried out without the aid of IOM.

KEYWORDS: Intraoperative neuromonitoring, Minimally invasive surgery, Oblique lateral interbody fusion

\section{INTRODUCTION}

$\mathrm{R}$ ecently, a new minimally invasive surgical technique using an oblique lateral corridor for lateral lumbar interbody fusion has been introduced $(1,2,5-7,12-$ $14,20)$. This technique, so-called "mini-open oblique lateral lumbar interbody fusion" (OLIF) exploits a window between the prevertebral venous structures and anterior border of the psoas muscle to access the targeted disc. Different from the traditional direct lateral lumbar approach passing through the psoas muscle, mini-open OLIF might reduce the complications related to lumbar plexus injury in the psoas muscle.
For the direct lateral lumbar interbody fusion (DLIF), the neurophysiological intraoperative monitoring (IOM) is mandatory to prevent lumbar plexus injury during the operation $(6,8,11,16)$. The guideline for mini-open OLIF also recommends the addition of IOM during surgery but there is doubt concerning the necessity of the IOM because of its clear trait that can treat psoas muscle minimally.

The purpose of this retrospective study was to verify the necessity of IOM in mini-open OLIF by comparing the clinical results and incidence of the transient and persistent 
perioperative complications between the patients who underwent mini-open OLIF with and without IOM.

\section{MATERIAL and METHODS}

From October 2013 to September 2015, total 129 consecutive patients who were diagnosed as degenerative spinal disease from L1 to S1 and underwent mini-open OLIF were identified and retrospectively reviewed. The operations were conducted by three different surgeons (K.S.R, J.S.K and J.W.H) with the same surgical protocol.

\section{Surgical Procedure}

Under general anesthesia, the patient was placed on the radiolucent table in the left lateral position. In the IOM group, only a fast-acting neuromuscular blocking agent was used for intubation. The IOM system (NIM-SPINE ${ }^{\mathrm{TM}}$ System, Medtronic, Memphis, TN, USA) was applied. Needle-recording electrodes were placed in each innervated muscle in the legs to monitor the affected nerve roots. In non-IOM group, all surgical procedures were performed without neuromonitoring. Under C-arm fluoroscopic guidance, a small skin incision of $2 \mathrm{~cm}$ was made in $6-8 \mathrm{~cm}$ in front of the center of the target disc. After the serial dissection of three layers of abdominal muscles, the retroperitoneal space was identified. The window between the inferior vena cava or left common iliac vein and anterior border of the psoas muscle was exposed. After confirming the annulus of the targeted disc, a guide pin was placed into the intervertebral disc space. A tubular retractor was docked after applying sequential serial dilators. The disc removal and endplate preparation were carried out by using shavers, curettes, and forceps. Finally, a cage (PEEK Clydesdale ${ }^{\circledR}$, Medtronic, Memphis, TN, USA) with the proper size was inserted. All procedures were performed by an orthogonal maneuver under C-arm fluoroscopic guidance. The layer-by-layer wound closure was done in the usual way.

All patients were followed up postoperatively using a predesigned protocol. According to the use of IOM, the patients were classified into two groups: the non-IOM group and the IOM group. The IOM group was mostly used at the first half of the study period, and the non-IOM group at the second half. An independent third party, an experienced clinical study coordinator who was blinded to all relevant knowledge of the patients, assessed the clinical outcomes using a visual analog pain scale (VAS) and the Oswestry disability index (ODI).

The perioperative complications were classified based on the relation to the surgical procedure and the effect of duration. According to the relation to the surgical procedure, the complications were divided into two groups; 'procedure-related' and 'procedure-unrelated'. Based on the effect of duration, the complications were defined as 'transient'; symptom relieved within 30 days postoperatively, and 'persistent'; symptom remains more than 30 days postoperatively. Perioperative complications until 3 months postoperatively were reviewed.

Numerical results were averaged. All numerical findings were expressed as means \pm SDs. The statistical program
SPSS for Windows version 11.0.1(SPSS Inc.) was used for the statistical calculations, and results were considered statistically significant for $p$ values $<0.05$

\section{RESULTS}

The study groups comprised 57 cases in the IOM group and 72 in the non-IOM group. Demographic data are shown in Table I. There were no significant statistical differences in perioperative parameters between the two groups, except the preoperative duration of the symptoms. The IOM group showed longer mean preoperative symptom duration (IOM; 33.6 months, non-IOM; 20.6 months). The IOM group required 20.4 minutes of mean preparation time for assembling the IOM before surgery. The distribution of the operated level is listed in Table II. Single level surgery was the most common in both groups, and the distribution of the number of levels was similar in both groups.

At 3 months after the operation, mean VAS scores of the IOM and non-IOM groups were significantly decreased compared to their preoperative values from $7.9 \pm 1.4$ to $2.7 \pm 1.8$ and $7.8 \pm 1.5$ to $2.6 \pm 1.4$, respectively $(p<0.005)$. Mean ODI scores of the IOM and non-IOM groups were also significantly decreased from $44.0 \pm 11.5$ to $15.4 \pm 8.9$ and $41.0 \pm 12.7$ to $14.1 \pm 7.8$, respectively $(p<0.05)$. There were no significant differences in clinical outcomes obtained in both groups (Figure 1).

The IOM group showed a $24.6 \%$ incidence of procedurerelated complications, and the non-IOM group 29.2\%, and these are summarized in Table III. Among the patients presenting with procedure-related complications in the IOM group, 10 patients $(17.6 \%)$ were classified as transient and $4(7.0 \%)$ as persistent complications. In the patients of the non-IOM group, 18 patients $(25.0 \%)$ were defined as transient and $3(4.2 \%)$ were persistent. Incidence of postoperative leg symptoms related to lumbar plexus and/or psoas muscle injury including pain, sensory and motor disturbance was shown as 6 transient and 3 persistent in the IOM group (overall $15.8 \%$ ), and 12 transient and 3 persistent in the non-IOM group (overall $20.9 \%$ ).

The overall incidence of procedure-unrelated complication accounted for $14.0 \%$ in the IOM group and $12.5 \%$ in the non-IOM group, respectively (Table IV). The incidence of the complications in each group is summarized in Table V.

In the IOM group, 5 patients $(8.8 \%)$ required re-operation because of bleeding, infection, pedicle screw malposition, and persistent pain. In the non-IOM group, 3 patients $(4.2 \%)$ needed re-operation for infection and ureteral injury.

\section{DISCUSSION}

The mini-open lateral access technique for lumbar interbody fusion has the unique benefits preserving the posterior of posterior spinal elements including the nervous structures, and feasibility of the placement of a larger cage enhances the fusion success rate and successful reduction of disc height. On the contrary, lateral access to the lumbar spine should manipulate psoas muscle and there is a risk of 


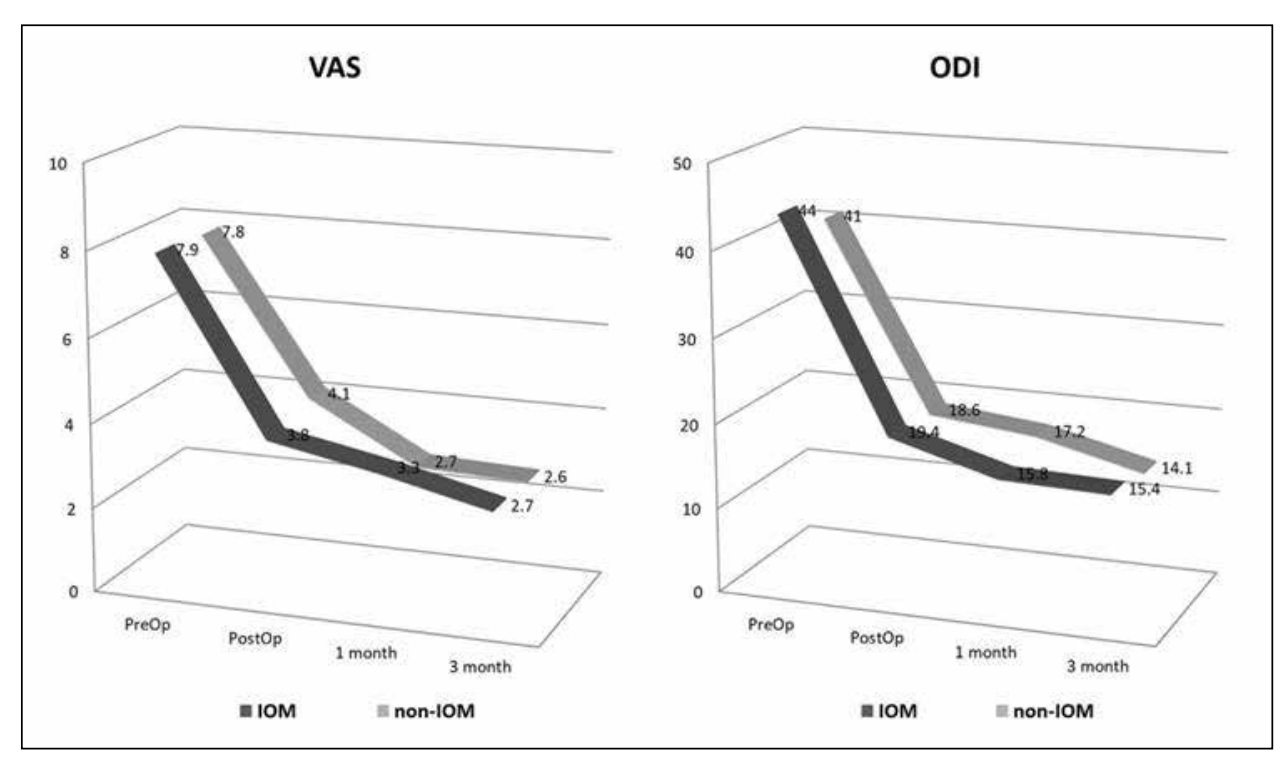

Figure 1: 3D Line graphs showing the clinical results according to the type of surgery.

Table I: Demographic Features of the Patients

\begin{tabular}{lccc}
\hline & IOM & Non-IOM & p value \\
\hline Number of cases & 57 & 72 & - \\
\hline Male:Female & $24: 33$ & $23: 49$ & $\mathrm{~N} / \mathrm{S}^{*}$ \\
\hline Age (years) & $65.6( \pm 8.8)$ & $67.1( \pm 9.5)$ & $\mathrm{N} / \mathrm{S}$ \\
\hline Duration of symptoms (months) & $33.6( \pm 24.9)$ & $20.6( \pm 12.1)$ & $<0.05$ \\
\hline Intraoperative bleeding amount (cc) & $120.2( \pm 34.2)$ & $108.2( \pm 32.4)$ & $\mathrm{N} / \mathrm{S}$ \\
\hline Preparation time for the assembling the IOM (minutes) & $20.4( \pm 11.2)$ & - & $\mathrm{N} / \mathrm{S}$ \\
\hline Operating time (minutes) & $62.6( \pm 22.2)$ & $60.5( \pm 23.3)$ & $\mathrm{N} / \mathrm{S}$ \\
\hline Length of hospital stay (days) & $14.6( \pm 13.4)$ & $12.1( \pm 7.1)$ & \\
\hline
\end{tabular}

*N/S: Non-specific, IOM: Intraoperative monitoring.

Table II: The Distribution of the Level of Surgery

\begin{tabular}{lcc}
\hline No. of levels & IOM & Non-IOM \\
\hline 1 & $24(42.1 \%)$ & $39(54.1 \%)$ \\
\hline 2 & $18(31.5 \%)$ & $21(29.1 \%)$ \\
\hline 3 & $14(24.5 \%)$ & $9(12.5 \%)$ \\
\hline 4 & $1(1.7 \%)$ & $2(2.7 \%)$ \\
\hline Total & 57 & 72 \\
\hline
\end{tabular}

IOM: Intraoperative monitoring.

lumbar plexus injury, especially in mini-open surgery using a tubular retractor. IOM is therefore mandatory to monitor and prevent lumbar plexus injury. On the other hand, many clinical series related to the lateral lumbar approach have reported frequent complications related to lumbar plexus injury with an incidence of $19 \%$ to $63 \%$, despite the routine use of IOM $(3,4,9,10,16,18,19)$. Even though most of those complications improved gradually, some patients were left with permanent sequelae.
Since first reported in 2012 (17), the oblique lateral approach to the lumbar spine has been developed in a minimally invasive manner by using a tubular retractor. In contrast with the traditional direct lateral lumbar approach, mini-open OLIF provides access to the target disc obliquely by entering a window between the prevertebral major vessels and the anterior border of psoas muscle. Because this approach does not pass through the psoas muscle, it is expected to reduce the complications related to lumbar plexus injury. Silvestre et al.(17) noted that only a $3.9 \%$ rate of complications related to lumbar plexus injury or psoas muscle weakness following OLIF in 179 patients. Sato et al.(15) also reported a 10\% incidence of leg complications after OLIF surgery in 20 patients with degenerative lumbar spondylolisthesis. Our previous report comparing the perioperative complications between DLIF and OLIF showed a $59.1 \%$ rate of approach-related complications in the DLIF group and $14.3 \%$ in the OLIF group (7). All those results demonstrate that oblique lateral access to the lumbar spine is much safer than direct lateral trans-psoas access regarding lumbar plexus or psoas muscle injury. 
There is a dilemma on whether neurophysiological IOM is necessary during mini-open OLIF surgery. It takes time to prepare and assemble the IOM. In addition, for successful monitoring, surgeon should mind various conditions such as careful anesthesia with proper anesthetics and muscle relaxants, minimal signal artifacts by electrical devices, and qualified interpretation of neurophysiological responses. In the present study, the IOM group required 20.4 minutes of mean preparation time for assembling the IOM before surgery. The mini-open OLIF intends minimal manipulation of the psoas muscle and lumbar plexus and it is possible that intentional neurophysiological monitoring will not be needed during the procedure. In the current study, the overall incidence of approach-side leg symptoms including pain and sensory and motor disturbance was $15.3 \%$ in the IOM group and $20.9 \%$ in the non-IOM group, with the non-IOM group having a higher

Table III: Procedure-Related Postoperative Complications

\begin{tabular}{|c|c|c|c|c|}
\hline & \multicolumn{2}{|c|}{ IOM (n=57) } & \multicolumn{2}{|c|}{ non-IOM (n=72) } \\
\hline & Transient & Persistent & Transient & Persistent \\
\hline Leg symptom & $6(10.5 \%)$ & $3(5.3 \%)$ & $12(16.7 \%)$ & $3(4.2 \%)$ \\
\hline Hip flexion weakness & 1 & - & 2 & - \\
\hline Hyperesthesia & 2 & 2 & 3 & 2 \\
\hline Dysesthesia & 1 & - & 2 & 1 \\
\hline Cold sensation & 2 & 1 & 3 & - \\
\hline Swelling & - & - & 2 & - \\
\hline Wound symptom & $4(7.0 \%)$ & $1(1.8 \%)$ & $6(8.3 \%)$ & - \\
\hline Infection & 2 & - & 5 & - \\
\hline Local hematoma & 2 & - & 1 & - \\
\hline Wound site pain & - & 1 & - & - \\
\hline Total & $10(17.6 \%)$ & $4(7.0 \%)$ & $18(25.0 \%)$ & $3(4.2 \%)$ \\
\hline
\end{tabular}

IOM: Intraoperative monitoring.

Table IV: Procedure-Unrelated Postoperative Complications

\begin{tabular}{lll}
\hline & IOM (n=57) & non-IOM (n=72) \\
\hline Abdominal ileus & $3(5.2 \%)$ & $2(2.7 \%)$ \\
\hline Pneumonia/Pleural effusion & $2(3.5 \%)$ & $1(1.3 \%)$ \\
\hline Urinary tract infection / acute renal failure & $3(5.2 \%)$ & $4(5.5 \%)$ \\
\hline Deep vein thrombosis & $0(0 \%)$ & $2(2.7 \%)$ \\
\hline Total & $8(14.0 \%)$ & $9(12.5 \%)$ \\
\hline
\end{tabular}

IOM: Intraoperative monitoring.

Table V: Summary of Postoperative Complications

\begin{tabular}{lcccc}
\hline & & IOM & non-IOM & p \\
\hline \multirow{2}{*}{ Procedure- related } & Transient & $10(17.6 \%)$ & $18(25.0 \%)$ & $<0.05$ \\
\cline { 2 - 5 } & Persistent & $4(7.0 \%)$ & $3(4.2 \%)$ & $\mathrm{N} / \mathrm{S}$ \\
\cline { 2 - 5 } Procedure-unrelated & Total & $14(24.5 \%)$ & $\mathrm{N} / \mathrm{S}$ & $\mathrm{N} / \mathrm{S}$ \\
\hline Total & & $8(14.0 \%)$ & $9(12.5 \%)$ & $\mathrm{N} / \mathrm{S}$ \\
\hline
\end{tabular}

IOM: Intraoperative monitoring, N/S: Non-specific. 
occurrence rate. However, the non-IOM group had a lower incidence of persistent leg symptoms at $4.2 \%$ (3 in 72 cases) compared to the IOM group at 5.3\% (3 in 57 cases). Moreover, the revision rate was $8.1 \%$ in the IOM group, and $5.1 \%$ in the non-IOM group. OILF without IOM could therefore increase the overall incidence of lumbar plexus or psoas muscle injury compared to OILF with IOM. However, the difference was not large and persistent complications were more frequent in cases of OLIF with IOM. Therefore, the current results indicate that IOM would not be essential in mini-open OLIF surgery.

Other major concerns of the OLIF would be injury of abdominal contents, and injury to the peritoneum, abdominal wall muscles, major vessels and ureter encountered during oblique lateral access. There was no case of complications related to the major vessel injury in the current study. However, three cases of local hematoma required revision surgery (IOM group; 2, non-IOM group; 1). Around the corridor of the OLIF, there are smaller tributaries of major vessels, which are very diverse regarding the number and the course of the branches in each patient. Therefore, all procedures should be carefully performed under clear vision to prevent and control branching vessel injury. The current study showed 5 cases of abdominal ileus (IOM group; 3, non-IOM group; 2), and all cases recovered early. Careful bowel preparation and postoperative supportive care to reduce abdominal ileus should be concerned.

The current study has several weaknesses. The number of cases is small, and it is a retrospective non-randomized study conducted at a single center.

\section{- CONCLUSION}

Mini-open OLIF can be carried out without IOM. Although the incidence of complications related to lumbar plexus or psoas muscle injury was slightly higher than with IOM use, there was no significant difference, and the advantages are that the time and effort spent for installing the device are not necessary and the operation time is reduced. A large case study with longer follow-up is needed.

\section{- REFERENCES}

1. Abbasi $\mathrm{H}$, Abbasi $\mathrm{A}$ : Oblique lateral lumbar interbody fusion (OLLIF): Technical notes and early results of a single surgeon comparative study. Cureus 7: e351, 2015

2. Abbasi H, Murphy CM: Economic performance of oblique lateral lumbar interbody fusion (OLLIF) with a focus on hospital throughput efficiency. Cureus 7: e292, 2015

3. Dominguez I, Luque R, Noriega M, Rey J, Alia J, MarcoMartinez F: Extreme lateral lumbar interbody fusion. Surgical technique, outcomes and complications after a minimum of one year follow-up. Rev Esp Cir Ortop Traumatol 61:8-18, 2017

4. Epstein NE: Non-neurological major complications of extreme lateral and related lumbar interbody fusion techniques. Surg Neurol Int 7: S656-S659, 2016
5. Fujibayashi S, Hynes RA, Otsuki B, Kimura H, Takemoto M, Matsuda S: Effect of indirect neural decompression through oblique lateral interbody fusion for degenerative lumbar disease. Spine (Phila Pa 1976) 40: E175-182, 2015

6. Gill JS, Nagda JV, Aner MM, Keel JC, Simopoulos TT: Contralateral oblique view is superior to the lateral view for lumbar epidural access. Pain Med 17: 839-850, 2016

7. Jin J, Ryu KS, Hur JW, Seong JH, Kim JS, Cho HJ: Comparative study of the difference of perioperative complication and radiologic results: MIS-DLIF (Minimally invasive direct lateral lumbar interbody fusion) versus MIS-OLIF (Minimally invasive oblique lateral lumbar interbody fusion). Clin Spine Surg 31(1):31-36,2018

8. Knight RQ, Schwaegler P, Hanscom D, Roh J: Direct lateral lumbar interbody fusion for degenerative conditions: Early complication profile. J Spinal Disord Tech 22: 34-37, 2009

9. Kueper J, Fantini GA, Walker BR, Aichmair A, Hughes AP: Incidence of vascular complications during lateral lumbar interbody fusion: An examination of the mini-open access technique. Eur Spine J 24:800-809, 2015

10. Kwon B, Kim DH: Lateral lumbar interbody fusion: Indications, outcomes, and complications. J Am Acad Orthop Surg 24: 96-105, 2016

11. Lee YS, Park SW, Kim YB: Direct lateral lumbar interbody fusion: Clinical and radiological outcomes. J Korean Neurosurg Soc 55: 248-254, 2014

12. Ohtori S, Mannoji C, Orita S, Yamauchi K, Eguchi Y, Ochiai N, Kishida S, Kuniyoshi K, Aoki Y, Nakamura J, Ishikawa T, Miyagi M, Kamoda H, Suzuki M, Kubota G, Sakuma Y, Oikawa Y, Inage K, Sainoh T, Sato J, Shiga Y, Abe K, Fujimoto K, Kanamoto H, Toyone T, Inoue G, Takahashi K: Mini-open anterior retroperitoneal lumbar interbody fusion: Oblique lateral interbody fusion for degenerated lumbar spinal kyphoscoliosis. Asian Spine J 9: 565-572, 2015

13. Ohtori S, Orita S, Yamauchi K, Eguchi Y, Ochiai N, Kishida S, Kuniyoshi K, Aoki Y, Nakamura J, Ishikawa T, Miyagi M, Kamoda H, Suzuki M, Kubota G, Sakuma Y, Oikawa Y, Inage K, Sainoh T, Sato J, Fujimoto K, Shiga Y, Abe K, Toyone T, Inoue G, Takahashi K: Mini-open anterior retroperitoneal lumbar interbody fusion: Oblique lateral interbody fusion for lumbar spinal degeneration disease. Yonsei Med J 56: 10511059, 2015

14. Orita $S$, Inage $K$, Sainoh $T$, Fujimoto $K$, Sato J, Shiga $Y$, Kanamoto $\mathrm{H}$, Abe K, Yamauchi K, Aoki Y, Nakamura J, Matsuura Y, Suzuki T, Kubota G, Eguchi Y, Terakado A, Takahashi K, Ohtori S: Lower lumbar segmental arteries can intersect over the intervertebral disc in the oblique lateral interbody fusion approach with a risk for arterial Injury: Radiological analysis of lumbar segmental arteries by using magnetic resonance imaging. Spine (Phila Pa 1976) 42: 135142,2017

15. Sato J, Ohtori S, Orita S, Yamauchi K, Eguchi Y, Ochiai N, Kuniyoshi K, Aoki Y, Nakamura J, Miyagi M, Suzuki M, Kubota G, Inage K, Sainoh T, Fujimoto K, Shiga Y, Abe K, Kanamoto $\mathrm{H}$, Inoue G, Takahashi K: Radiographic evaluation of indirect decompression of mini-open anterior retroperitoneal lumbar interbody fusion: Oblique lateral interbody fusion for degenerated lumbar spondylolisthesis. Eur Spine J 26(3): 671-678, 2017 
16. Sedra F, Lee R, Dominguez I, Wilson L: Neurological complications using a novel retractor system for direct lateral minimally invasive lumbar interbody fusion. J Clin Neurosci 31: 81-87, 2016

17. Silvestre C, Mac-Thiong JM, Hilmi R, Roussouly P: Complications and morbidities of mini-open anterior retroperitoneal lumbar interbody fusion: Oblique lumbar interbody fusion in 179 patients. Asian Spine J 6: 89-97, 2012

18. Sofianos DA, Briseno MR, Abrams J, Patel AA: Complications of the lateral transpsoas approach for lumbar interbody arthrodesis: A case series and literature review. Clin Orthop Relat Res 470: 1621-1632, 2012
19. Waddell B, Briski D, Qadir R, Godoy G, Houston AH, Rudman E, Zavatsky J: Lateral lumbar interbody fusion for the correction of spondylolisthesis and adult degenerative scoliosis in high-risk patients: Early radiographic results and complications. Ochsner J 14: 23-31, 2014

20. Wakita H, Shiga Y, Ohtori S, Kubota G, Inage K, Sainoh T, Sato J, Fujimoto K, Yamauchi K, Nakamura J, Takahashi K, Toyone T, Aoki Y, Inoue G, Miyagi M, Orita S: Less invasive corrective surgery using oblique lateral interbody fusion (OLIF) including L5-S1 fusion for severe lumbar kyphoscoliosis due to L4 compression fracture in a patient with Parkinson's disease: A case report. BMC Res Notes 8: 126, 2015 